\title{
Histological Evaluation of Pulpal Response to Direct Pulp Capping with Biodentine and Mineral Trioxide Aggregate: An In Vivo Study
}

\author{
Mahendran Kavitha ${ }^{1}$, Hema J Christa ${ }^{2}$, Nandhini Ambalavanan ${ }^{3}$, Shakthi Priya Sivaprakasam ${ }^{4}$
}

\begin{abstract}
Aim: The aim of the present study is to compare the pulpal response to direct pulp capping with the mineral trioxide aggregate (MTA) and biodentine by light microscopic histological evaluation.

Materials and methods: A total of 45 premolars scheduled for orthodontic extraction were selected and subjected to the direct pulp capping procedure. Class I cavities were prepared and MTA or biodentine was placed over the exposed pulp, followed by a composite restoration. After the experimental periods of 7, 30, and 90 days, the teeth were extracted and histological processing was carried out. The test materials were evaluated histologically for the degree of inflammation, dentin bridge formation, and thickness of dentin bridge at all the three different observation periods, tabulated and statistically analyzed using the Chi-square test.

Results: At 7 days' observation, all the samples in groups I and II showed a mild inflammatory response. At 1 month, a thin or partial dentin bridge was evident in all the samples in both groups. At 3 months, all the samples in both groups showed evidence of complete dentin bridge formation. There was no statistically significant difference between the MTA and biodentine groups.

Conclusion: Biodentine was as effective as MTA in inducing a pulpal reaction with minimal inflammation and in dentin bridge formation.

Keywords: Biodentine, Dentin bridge, Direct pulp capping, Inflammation, Mineral trioxide aggregate, Orthodontic extraction.

Journal of Operative Dentistry and Endodontics (2019): 10.5005/jp-journals-10047-0080
\end{abstract}

\section{INTRODUCTION}

Direct pulp capping is the treatment of an exposed vital pulp by sealing the pulpal wound with a dental material placed directly on a mechanical or traumatic exposure to facilitate the formation of the reparative dentin and maintenance of the vital pulp. ${ }^{1}$ The intention is to maintain the pulp vitality and postpone more aggressive therapies that could eventually lower the long-term prognosis of the tooth.

A number of factors have been shown to have an impact on the success of direct pulp capping. An ideal pulp capping agent should stimulate reparative dentin formation, not affect pulpal vitality, not unleash any adverse effects when used, and have good sealing ability. ${ }^{2}$

Numerous materials have been used for pulp capping. One such time-tested material is calcium hydroxide. But the pulp tissue subjacent to calcium hydroxide is usually completely deranged and distorted, forming a zone of obliteration, its dissolution over time may lead to microleakage and the dentinal bridge formed is porous with "tunnel defects." ${ }^{3}$ Because of the above-mentioned disadvantages of calcium hydroxide, a variety of materials like mineral trioxide aggregate (MTA) and biodentine have been developed, tried, and tested for direct pulp capping.

The MTA has demonstrated a superior ability to maintain the integrity of the pulp tissue and produce a thicker and less porous dentinal bridge at a faster rate compared to calcium hydroxide. In addition, MTA is able to decrease pulp inflammation and presents significantly less toxicity and pulpal necrosis, which is statistically significant compared with $\mathrm{Ca}(\mathrm{OH})_{2}$.

Biodentine can be used as a dentin substitute on crowns and roots similar to MTA but with several added advantages like costeffectiveness, better handling properties, and reduced setting time.

\footnotetext{
${ }^{1-4}$ Department of Conservative Dentistry and Endodontics, Tamil Nadu Government Dental College and Hospital, Chennai, Tamil Nadu, India

Corresponding Author: Shakthi Priya Sivaprakasam, Department of Conservative Dentistry and Endodontics, Tamil Nadu Government Dental College and Hospital, Chennai, Tamil Nadu, India, Phone: +91 9677247520, e-mail: shakthiviolen@gmail.com

How to cite this article: Kavitha M, Christa HJ, Ambalavanan N, et al. Histological Evaluation of Pulpal Response to Direct Pulp Capping with Biodentine and Mineral Trioxide Aggregate: An In Vivo Study. J Oper Dent Endod 2019;4(2):57-61.

Source of support: Nil

Conflict of interest: None
}

Histological studies using biodentine as a pulp capping material have shown promising results. ${ }^{4,5}$

However there are limited in vivo studies using biodentine. Hence, in this study an attempt has been made to histologically compare the effects of biodentine (Septodont Saint-Maur-desFosses, France) and MTA (Angelus, Londrina, PR, Brazil) as direct pulp capping agents on human teeth.

\section{Materials and Methods}

This study was conducted at Tamil Nadu Government Dental College and Hospital after obtaining the approval from the Institutional Ethical Committee (7/IRB/2017). Young adult patients (age group: 18-24 years), scheduled for extraction of premolars for orthodontic reasons, were selected for the study. An informed consent was obtained from the patients. A total of 45 first or second human premolar teeth from 14 patients were selected. 
Seven patients contributed all four premolars each, four other patients contributed three teeth each, two of them gave consent for two teeth, and one patient contributed one tooth. All teeth were examined clinically and radiographically to ensure absence of caries and periapical pathosis.

Decayed, restored, or fractured teeth; teeth that were symptomatic or with periapical pathology; or those not conductive to rubber dam isolation were excluded from the study.

\section{Experimental Groups}

- Group I: MTA $(n=21)$

- Group II: Biodentine $(n=21)$

- Group III: Control-normal teeth $(n=3)$

\section{Experimental Protocol}

After administering the local anesthetic (2\% lignocaine) and rubber dam isolation, the teeth were cleaned with a rubber cup and prophylactic paste at low speed. The site was then washed with $2 \%$ chlorhexidine. Occlusal cavities of dimensions approximately $2 \mathrm{~mm}$ long, $2 \mathrm{~mm}$ wide, and of variable depth about $3.5 \mathrm{~mm}$ till pulp exposure was prepared using a sterile straight fissure diamond (Mani, Japan) at high speed under water spray coolant. The cavity walls were irrigated with normal saline. Then a pulp exposure was made using a no- 2 round $(0.9$ $\mathrm{mm}$ diameter) tungsten carbide bur (Mani, Japan) at high speed without penetrating the pulp space.

Hemorrhage was controlled by a sterile cotton pellet soaked in saline. Then, the area of pulp exposure was capped with MTA (Angelus, Londrina, PR, Brazil) or biodentine (Septodont SaintMaur-des-Fosses, France). The materials were mixed according to the manufacturer's instructions. A layer of flowable composite resin (Charmfil Flow/Nexcomp Flow, Meta Biomed) was then placed over the capping material. The cavity was then etched, rinsed, and filled with a nanofilled composite (Ivoclar Vivadent, Liechtenstein, Europe) resin.

All the experimental teeth were carefully followed up and assessed. The assessment included history taking and a clinical examination for recording any signs or symptoms and response to electric pulp testing and cold testing. The extractions were scheduled after a period of 1 week, 1 month, and 3 months. A total of 15 teeth were extracted at 1 week, another 15 teeth at 1 month, and another 15 teeth at 3 month interval. The teeth were grouped according to the timing of extraction and the pulp capping material used.

After atraumatic extraction, the teeth were fixed with $10 \%$ formalin for 96 hours, decalcified in 5\% nitric acid, and dehydrated using increasing concentrations of aqueous ethanol and embedded in paraffin. Then serial buccolingual sections of 4 micron thickness were cut longitudinally through the center of the exposure site stained and observed under a microscope (Olympus DX 4 SF). The sections were then blindly evaluated by an experienced pathologist and calibrated according to the criteria given in Table 1.

\section{Results}

No apparent adverse events were observed during the experimental periods and no dropouts were observed. The scores for inflammatory cell response, hard tissue formation, and thickness of dentinal bridge for the two experimental groups in all the observation periods were depicted in form of a graph (Fig. 1).
Table 1: Scoring criteria for histopathological evaluation

\begin{tabular}{|c|c|}
\hline Grading & Characterization \\
\hline Score & Inflammatory cell response \\
\hline 1 & $\begin{array}{l}\text { None or few scattered inflammatory cells present in } \\
\text { the site of pulp exposure, characteristic of normal } \\
\text { tissue }\end{array}$ \\
\hline 2 & $\begin{array}{l}\text { Mild inflammatory cell infiltration with PMN or } \\
\text { mononuclear }\end{array}$ \\
\hline 3 & $\begin{array}{l}\text { Moderate inflammatory cell infiltration involving } \\
\text { coronal pulp ( } 10-25 \text { cells })\end{array}$ \\
\hline 4 & $\begin{array}{l}\text { Severe inflammatory cell infiltration ( }>25 \text { cells) } \\
\text { involving coronal pulp or abscess present }\end{array}$ \\
\hline Score & Hard tissue formation \\
\hline 1 & Absent \\
\hline 2 & $\begin{array}{l}\text { Lateral deposition of the hard tissue on the walls of } \\
\text { the cavity of pulp exposure }\end{array}$ \\
\hline 3 & $\begin{array}{l}\text { Partial hard tissue bridge-little communication of the } \\
\text { capping material with pulp }\end{array}$ \\
\hline 4 & $\begin{array}{l}\text { Complete hard tissue bridge-closure of exposure } \\
\text { area }\end{array}$ \\
\hline Score & Thickness of dentinal bridge \\
\hline 1 & $0 \mathrm{~mm}$ \\
\hline 2 & $<0.1 \mathrm{~mm}$ \\
\hline 3 & $0.1-0.25 \mathrm{~mm}$ \\
\hline 4 & $>0.25 \mathrm{~mm}$ \\
\hline
\end{tabular}

The results were analyzed using the chi-square test using the Statistical Package for Social Sciences Version 16 (SPSS 16) software.

In the 7 days' samples, groups I and II showed mild or no inflammatory response (Figs $2 \mathrm{~A}$ and $\mathrm{B}$ ). The 1 month samples showed scores of 2 and 3 for formation of the dentin bridge and thickness of the dentin bridge. The score corresponding to inflammation was 1 in both groups as no inflammation was seen (Figs $2 \mathrm{C}$ and D). The 3 month samples showed scores of 3 and 4 for formation of the dentin bridge and thickness of the dentin bridge. The score corresponding to inflammation remained 1 as in the 1 month samples (Figs $2 \mathrm{E}$ and $\mathrm{F}$ and Table 2).

The results showed that there is no statistically significant difference between the groups in terms of inflammation, dentin bridge formation, and the thickness of the dentin bridge formed as the $p$ value calculated for each of the groups was above 0.05 .

\section{Discussion}

Pulp capping refers to a vital pulp therapy procedure designed to preserve and maintain pulpal vitality in teeth that have been exposed by trauma, caries, or restorative procedures. Calcium hydroxide has been considered the gold standard for pulp capping. The MTA is a calcium silicate-based cement developed by Torabinejad et al. in 1990. It is composed of tricalcium silicate, tricalcium aluminate, tricalcium oxide, silicate oxide, and bismuth oxide as a radio opacifier.

In a long-term clinical study done by Mente et al. ${ }^{6}$ to compare calcium hydroxide and MTA as direct pulp capping agents in carious teeth, it was concluded that with time, teeth in the calcium hydroxide group started becoming nonvital. However there was no time-dependent decline in the success rate when MTA was used 


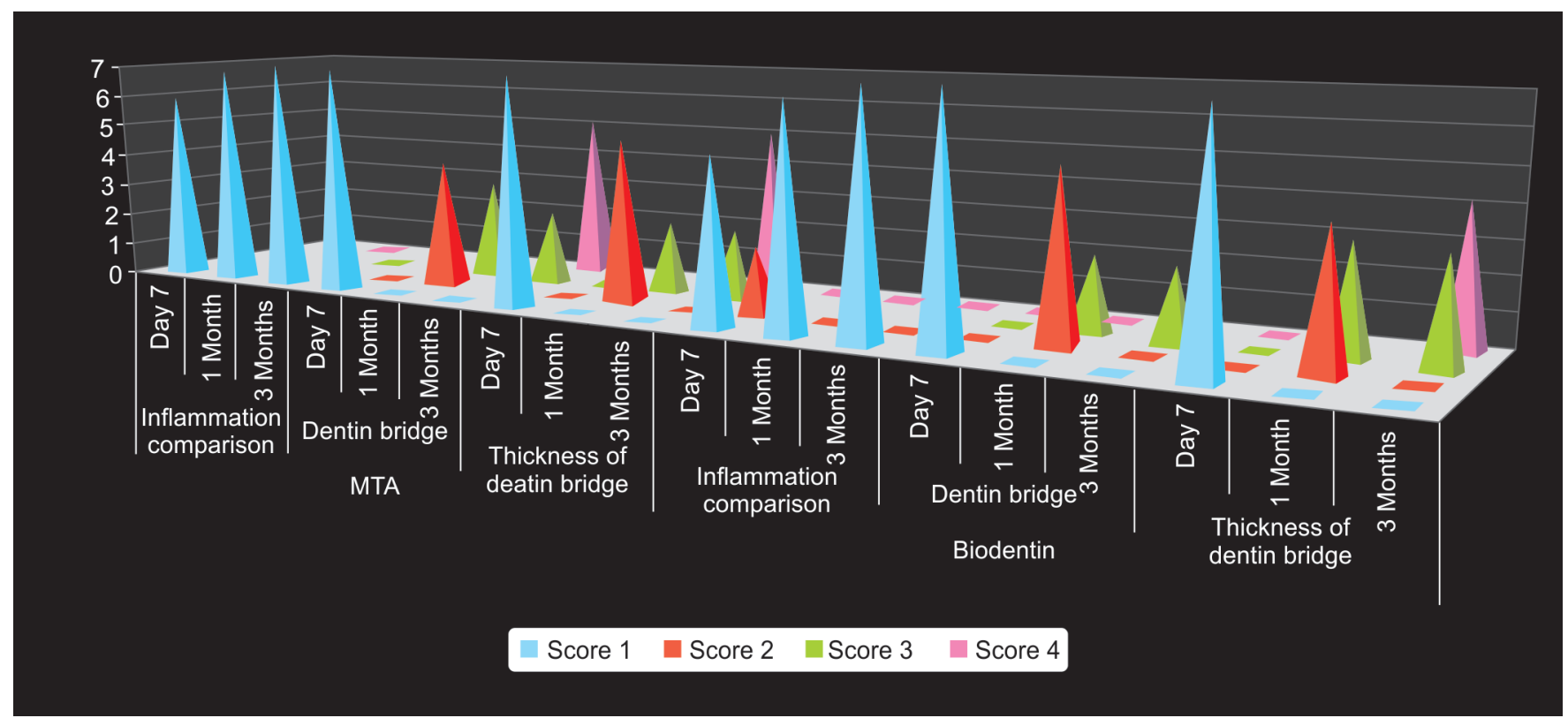

Fig. 1: Comparison of all the three parameters between MTA and biodentine groups at 7 days, 1 month and 3 months
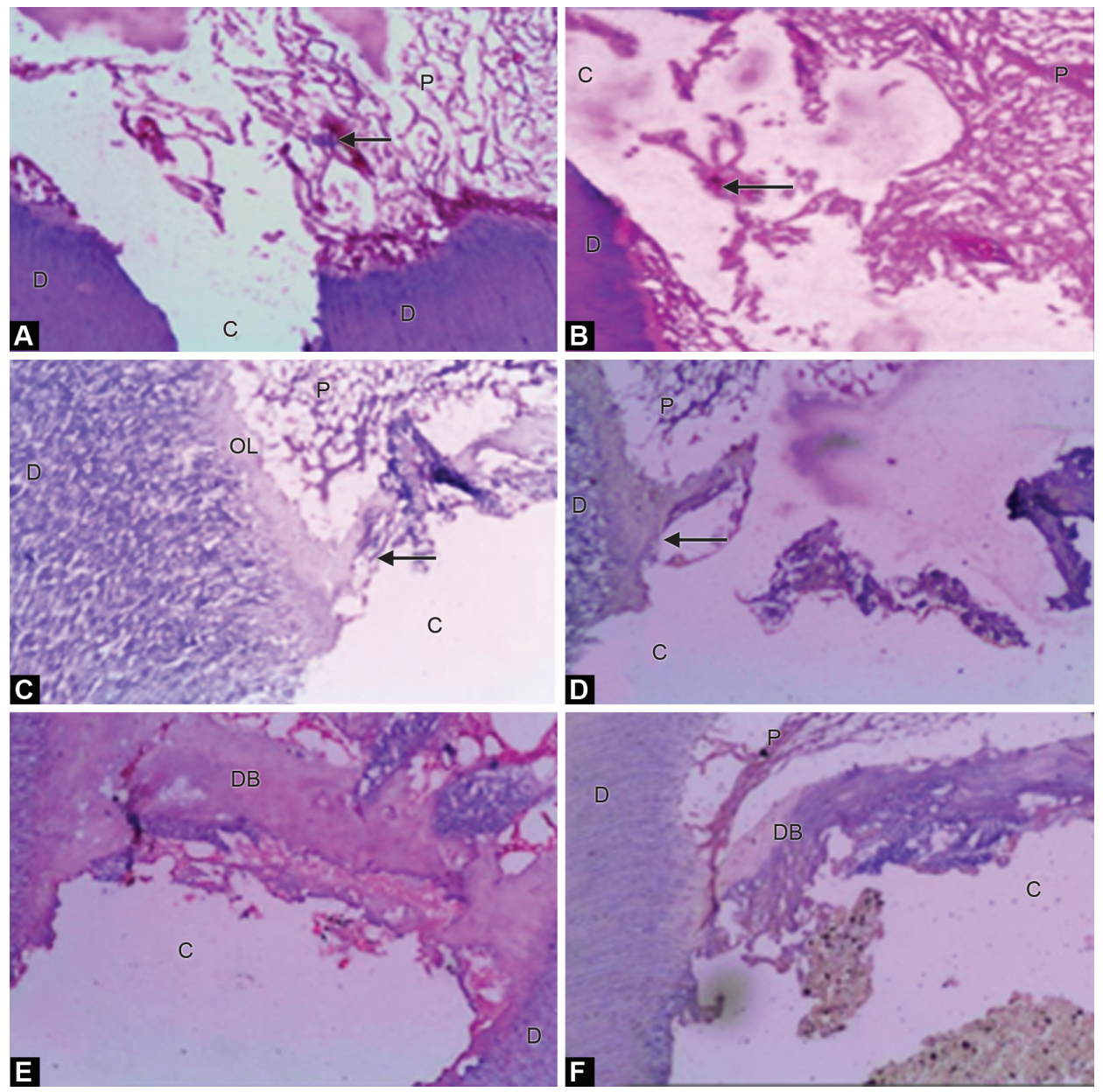

Figs 2A to F: Histologic image of pulpal response to: (A) MTA at 7 days. Mild inflammation was observed at the pulp (P) dentin (D) interface; (B) Biodentine at 7 days. Mild inflammation was observed at the pulp (P) dentin (D) interface; (C) MTA at 1 month. Thin dentin bridge (DB) formation was observed at the pulp (P) dentin (D) interface; (D) Biodentine at 1 month. Thin dentin bridge formation was observed at the pulp (P) dentin (D) interface; (E) MTA at 3 months. Well-formed dentin bridge could be appreciated at the pulp (P) dentin (D) interface; (F) MTA at 3 months. Wellformed dentin bridge could be appreciated at the pulp (P) dentin (D) interface 
Comparison of MTA and Biodentine as Direct Pulp Capping Agents

Table 2: Histopathological scores and Chi-square statistics MTA (at 3 months)

\begin{tabular}{lllll}
\hline Histological criteria & Score & MTA $(n=7)$ & $B D(n=7)$ & $p$ value \\
\hline Hard tissue formation at 3 months & 3-Partial hard tissue bridge & 2 & 2 & 1.00 (NS) \\
& 4-Complete hard tissue bridge & 5 & 5 & \\
Dentin bridge thickness at 3 & $3-(0.1-0.25 \mathrm{~mm})$ & 2 & 3 & 0.577 (NS) \\
months & $4-(>0.25 \mathrm{~mm})$ & 5 & 4 & \\
Inflammation at 3 months & 1 & 7 & 7 & (NS) \\
\hline
\end{tabular}

as a direct pulp capping agent. This time-dependent decrease in success rate on calcium hydroxide usage has been observed in many clinical studies. This can be attributed to the various drawbacks of calcium hydroxide like poor bonding to dentin, material resorption, and mechanical instability.

In another study by Aeinehchi et al. ${ }^{7}$ comparing the use of MTA and calcium hydroxide in direct pulp capping cases, odontoblastic layers appeared earlier; less hyperemia, inflammation, and necrosis were noted; and dentinal bridges were more pronounced in the MTA-treated teeth. Similar results have been drawn by Nair et al. ${ }^{8}$ comparing MTA and calcium hydroxide.

Compared to MTA, biodentine has better handling properties with a homogeneous consistency similar to phosphate cement, with a controlled setting time of 9-12 minutes and is also is costeffective when compared to MTA.

To our knowledge, only a few clinical investigations have compared biodentine and MTA in humans. ${ }^{9}$ This study presents a light microscopic analysis $(10 X)$ of the pulpal response to direct pulp capping, with biodentine and MTA in healthy human premolars, which were scheduled for extraction due to orthodontic reasons.

We have used a fast setting version of MTA, namely MTA (Angelus), which has a setting time of 15 minutes. This was done to achieve standardization of both the pulp capping materials. The pulp capping material MTA/BD was stabilized with a thin layer of flowable composite and immediately sealed with a light-cure composite restoration after etching and bonding. ${ }^{10}$ This procedure was done to prevent microleakage.

In our study, a period of 1 week, 1 month, and 3 months was used to evaluate the pulp response to capping materials. In 7 days, both the groups showed mild or no inflammatory response with very few inflammatory cells infiltrating the exposed area.

In 30 days, a reduction in the inflammatory response and formation of a thin or partial dentin bridge was evident in both groups. This represents a positive healing outcome.

After 90 days, both groups showed evidence of complete dentin bridge formation closing the exposed area. There was no significant difference between the groups in all the three criteria.

The MTA induced an early form of reparative dentin synthesis, probably because of modulation of pulp cell transforming growth factor-beta 1 secretion. ${ }^{11,12}$ Direct pulp capping with biodentine also produced a similar response. Similarity of the tissue response with these materials may be due to their similar chemical composition (tricalcium silicate), the by-product released during setting reaction, and physical properties. ${ }^{13}$

A study ${ }^{14}$ showed that particles of biodentine were entrapped in the newly formed foci, and mineralization appeared as osteodentin. Stimulated cellular proliferation and differentiation might be due to the presence of tricalcium silicate, one of the main components of biodentine, and the presence of both calcium and silicon ions. ${ }^{11,15-17}$

Tran et al. ${ }^{15}$ compared biodentine to calcium hydroxide and MTA in a rat pulp injury model. At day 7 , results showed that both biodentine and MTA induced cell proliferation and formation of a homogeneous dentin bridge at the injury site, which was strongly positive for osteodentin. In contrast, the reparative tissue induced by calcium hydroxide showed porous organization. ${ }^{18}$

Since the capping procedure was accomplished in sound teeth, the results of this study should be carefully evaluated. In most clinical scenarios, the pulp exposure frequently occurs by a carious process during which the level of inflammation is much higher. Therefore, the relevance of these studies conducted in healthy human teeth could be clinically limited, and further long-term assessment is required to evaluate the pulp response to biodentine in the inflamed pulp.

Also, additional studies are needed with immunohistochemistry and transmission electron microscopy to assess the mode of action of biodentine on the pulp.

\section{Conclusion}

Within the limitations of this study, we can conclude that there is no statistically significant difference in the inflammatory process and formation of the dentin bridge between MTA and biodentine and that the pulp healing potential of biodentine is comparable to that of MTA. Further long-term clinical trials are required to evaluate the effect of biodentine as a pulp capping material.

\section{Clinical Relevance Statement}

Biodentine was as effective as the MTA in the pulp capping procedure. It also has comparatively better handling properties than MTA, therefore is a promising alternative to MTA for direct pulp capping procedures.

\section{References}

1. Glossary of Endodontic terms. 7th edn, Chicago (IL): American Association of Endodontists; 2003.

2. Wataha JC. Biocompatibility of dental materials. In: Craig RG, Powers JM. Restorative dental materials. St. Louis: Mosby; 2002. 125-162.

3. Cox CF, Subay RK, Ostro E, et al. Tunnel defects in dentin bridges: their formation following direct pulp capping. Oper Dent 1996;21(1):4-11.

4. Dammaschke T, Wolff $P$, Sagheri $D$, et al. Mineral trioxide aggregate for direct pulp capping: a histologic comparison with calcium hydroxide in rat molars. Quintessence Int 2010;41(2):20-30.

5. Zarrabi $M H$, Javidi $M$, Jafarian $A H$, et al. Histologic assessment of human pulp response to capping with mineral trioxide aggregate and a novel endodontic cement. J Endod 2010;36(11):1778-1781. DOI: 10.1016/j.joen.2010.08.024.

6. Mente J, Geletneky B, Ohle M. Mineral trioxide aggregate or calcium hydroxide direct pulp capping: an analysis of the clinical treatment outcome. J Endod 2010;36(5):806-813. DOI: 10.1016/ j.joen.2010.02.024

7. Aeinehchi M, Eslami B, Ghanbariha M, et al. Mineral trioxide aggregate (MTA) and calcium hydroxide as pulp_capping agents in human teeth: a preliminary report. Int Endod J 2003;36(3):225-231. DOI: 10.1046/j.1365-2591.2003.00652.x. 
8. Nair PN, Duncan HF, Pitt Ford TR, et al. Histological, ultrastructural and quantitative investigations on the response of healthy human pulps to experimental capping with mineral trioxide aggregate: a randomized controlled trial. Int Endod J 2008;41(2):128-150.

9. Hegde S, Sowmya B, Mathew S, et al. Clinical evaluation of mineral trioxide aggregate and biodentine as direct pulp capping agents in carious teeth. J Conserv Dent 2017;20(2):91-95. DOI: 10.4103/09720707.212243.

10. Masaki T, Yasuhisa T, Atsushi $\mathrm{O}$, et al. Timing for composite resin placement on mineral trioxide aggregate. J Endod 2013;39(9): 116-1170.

11. Yasuda $Y$, Ogawa M, Arakawa T, et al. The effect of mineral trioxide aggregate on the mineralization ability of rat dental pulp cells: an in Vitro study. J Endod 2008;34(9):1057-1060. DOI: 10.1016/ j.joen.2008.06.007.

12. Seo $M$, Hwang $K$, Lee $J$. The effect of mineral trioxide aggregate on Odontogenic differentiation in dental pulp stem cells. J Endod 2013;39(2):242-248. DOI: 10.1016/j.joen.2012.11.004.
13. Zhou HM, Shen Y, Wang ZJ, et al. In vitro cytotoxicity evaluation of a novel root repair material. J Endod 2013;39:478-483. DOI: 10.1016/ j.joen.2012.11.026.

14. Laurent $P$, Camps J, De Méo $M$, et al. Induction of specific cell responses to a $\mathrm{Ca}_{3} \mathrm{SiO}_{5}$-based posterior restorative material. Dent Mater 2008;24(11):1486-1494. DOI: 10.1016/j.dental.2008.02.020.

15. Tran XV, Gorin C, Willig C, et al. Effect of a calcium-silicate-based restorative cement on pulp repair. J Dent Res 2012;91:1166-1171. DOI: $10.1177 / 0022034512460833$.

16. Peng W, Liu W, Zhai W, et al. Effect of tricalcium silicate on the proliferation and odontogenic differentiation of human dental pulp cells. J Endod 2011;37(9):1240-1246. DOI: 10.1016/j.joen.2011.05.035.

17. Lipski M, Nowicka A, Kot K, et al. Factors affecting the outcomes of direct pulp capping using biodentine. Clin Oral Investig 2018;22(5):2021-2029. DOI: 10.1007/s00784-017-2296-7.

18. Jalan $A L$, Warhadpande MM, Dakshindas DM. A comparison of human dental pulp response to calcium hydroxide and biodentine as direct pulp-capping agents. J Conserv Dent 2017;20(2):129-133. DOI: 10.4103/0972-0707.212247. 